\title{
ULAM-ZAHORSKI PROBLEM ON FREE INTERPOLATION BY SMOOTH FUNCTIONS
}

\author{
A. OLEVSKIII
}

\begin{abstract}
Let $f$ be a function belonging to $C^{n}[0,1]$. Is it possible to find a smoother function $g \in C^{n+1}$ (or at least $C^{n+\varepsilon}$ ) which has infinitely many points of contact of maximal order $n$ with $f$ (or at least arbitrarily many such points with fixed norm $\left.\|g\|_{C^{n+\varepsilon}}\right)$ ? It turns out that for $n=0$ and 1 the answer is positive, but if $n \geq 2$, it is negative. This gives a complete solution to the Ulam-Zahorski question on free interpolation on perfect sets.
\end{abstract}

\section{INTRODUCTION}

In this paper we consider the following problem: is it possible for a given smooth function $f$ defined on the segment $I=[0,1]$ to find another smoother function $g$ which coincides with $f$ on some (nonempty) perfect set? Of course, this set has not been fixed, its choice is dependent on $f$, so we are dealing with free interpolation.

The question was first posed by Ulam, who conjectured in the mid-thirties that "for every $f \in C(I)$ there exists an analytic function $g$ which coincides with $f$ on some perfect set" (see [U, Sc]).

One can observe that if $f$ is "bad", say nowhere differentiable, then some level sets are uncountable and we can put $g \equiv$ const .

However, the conjecture was disproved by Zahorski who has constructed the example of a function $f \in C^{\infty}(I)$ for which the radius of convergence of its Taylor expansion at every point $x$ is equal to zero [Z]. It is clear that for analytic $g$ the set $\{t: f(t)=g(t)\}$ consists of only isolated points. It should be mentioned that the first example of this kind was discovered by $\mathrm{H}$. Cartan [Ca]. After giving a counterexample, in the same paper, Zahorski (1947) asked the following variation of the problem:

"It is true that there exists a function $f \in C(I)$ such that for every $g \in$ $C^{\infty}(I)$, or $C^{p}(I)$, the set $E_{f, g}=\{t: f(t)=g(t)\}$ is at most countable?"

In recent years this topic has been interestingly developed in a number of papers [ABLP, $\mathrm{La}, \mathrm{Ag}, \mathrm{Br}, \mathrm{Bu}$ ]. Laczkovich proved [La] that for every $f \in C(I)$ there is a perfect set $E \subset I$ such that $\left.f\right|_{E} \in C^{\infty}(E)$. Argonski, Bruckner, Laczkovich, and Preiss showed that every $f \in C(I)$ can be interpolated on some perfect set by a $g \in C^{1}(I)$ [ABLP] (1985). The same authors also proved that for every $f \in C(I)$ one can find $g \in C^{\infty}(I)$ such that $E_{f, g}$ is an infinite

Received by the editors May 18, 1992.

1991 Mathematics Subject Classification. Primary 26A48, 26A51. 


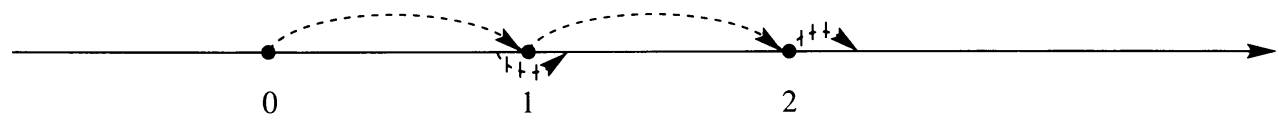

FIGURE 1

set. But they noted that Zahorski's problem for $g \in C^{2}$ (or $C^{\infty}$ ) remained open.

In 1988 , Buczolich presented a result close to the case $g \in C^{2}$. He proved [Bu] that every $f \in C(I)$ can be interpolated (on some perfect set) by a convex function $g$.

Now we state the main results of this paper.

1. Zahorski's problem for $p \geq 2$ has a positive solution. Moreover, in Theorem 1 we prove that there exists a Lipschitz function $f$ such that for every $g \in C^{1+\varepsilon}(I)(\varepsilon>0)$ the set $E_{f, g}$ is at most countable.

In this paper we denote by $C^{\alpha}(I), 0<\alpha<1$, the Hölder class of order $\alpha$. If $\alpha>1$ and not an integer, $f \in C^{\alpha}(I)$ means that $f \in C^{k}, k=[\alpha]$, and $f^{(k)} \in C^{\alpha-k}$. We have the following picture: if the smoothness of $f$ is equal to zero, then, by free interpolation on a perfect set, we can improve the order of smoothness to 1 [ABLP]. But even if the smoothness of $f$ is close to 1 we cannot, in general, achieve smoothness $1+\varepsilon$ for $g$.

It is impossible to jump over 1 .

This effect occurs in the neighborhood of every integer: namely if $f \in C^{n-\varepsilon}$ is general, it cannot be interpolated (on a perfect set) by $g \in C^{n+\varepsilon}$. To show this it will be enough to integrate the function in Theorem $1 n-1$ times. One can expect (keeping in mind fractional integration, for example) that the movement for $\alpha \geq 1$ is impossible. But this is not so.

2. The following statement is verified in Theorem 2: For every $f \in C^{1}(I)$ there exists $g \in C^{2}(I)$ which coincides with $f$ on some perfect set.

So in Figure 1 the jump from 1 to 2 is possible.

3. Let us look at $\alpha=2$, that is $f \in C^{2}(I)$. One should expect that $f$ can be interpolated by a $g \in C^{3}(I)$. It seems surprising that it cannot be done.

There exists a function $f \in C^{2}(I)$ which cannot be interpolated on any perfect set by any $g \in C^{3}$ (or even by $g \in C^{2+\varepsilon}$ ) (Theorem 4).

The result holds for every $\alpha \geq 2$. If we remain at this point movement to the right is impossible.

We mention that the phenomenon can be stated in a simple way by avoiding the analysis of cardinality. Indeed the following corollary of Theorem 3 is true:

There exists $f \in C^{2}(I)$ such that for every $g \in C^{3}(I)$ there is only finitely many, say $\nu$, points of contact of order 2. The estimate of $\nu$ depends only on $\|g\|_{C^{3}}$.

This contrasts with the case of small $n$ : for every $f \in C^{n}(n=0,1)$ some $g \in C^{n+1}$ has contact of maximal order with $f$ at infinitely many points.

The main results of this paper were presented the first time by the author at the Symposium on Real Analysis in San Bernardino (June 1990) [Ol].

\section{1}

Theorem 1. There exists a Lipschitz function $f: I=[0,1] \rightarrow \mathbb{R}$ such that for every $g \in C^{\gamma}(I), \gamma>1$, the set $E_{f, g}$ is at most countable with only a finite set of accumulation points. 
Proof. Let $r(t)$ be a "step function": $r(t)=\operatorname{sign} t,|t|<1$, which is extended periodically on $\mathbb{R}$. Suppose

$$
f_{1}(t)=\sum q^{k} r\left(2^{\nu_{k}} t\right), \quad f(t)=\int_{0}^{t} f_{1}(\tau) d \tau .
$$

We prove that if

$$
0<q<1 / 10 ; \quad k=o\left(\nu_{k}\right), \quad \nu_{1}<\nu_{2}<\cdots,
$$

then $f$ satisfies the statement of the theorem. It is clear that $f$ is Lipschitz. It is easy to see that $f_{1}$ has one-sided limits at every point, so $f$ has one-sided derivatives:

$$
f_{+}^{\prime}(s)=f_{1}(x+0) ; \quad f_{-}^{\prime}(x)=f_{1}(x-0) .
$$

Let $g \in C^{\gamma}(I)$ for some $\gamma=1+\varepsilon, \varepsilon>0$, and $E^{-}$be the set of left-hand accumulation points of the set $E_{f, g}$ (so $x>0$ belongs to $E^{-}$if it is a limit of any increasing sequence $\left.t_{n} \in E_{f, g}\right)$. Then

$$
g(x)=f(x), \quad g^{\prime}(x)=f_{1}(x-0) \quad \forall x \in E^{-} .
$$

Intervals $\left.\Delta=] j \cdot 2^{-\nu_{k}},(j+1) 2^{-\nu_{k}}\right]\left(j=0,1, \ldots, 2^{\nu_{k}}-1\right)$ we call intervals of range $k$. We show that if $k_{0}$ is large enough every interval $\Delta$ of range $k_{0}$ contains at most one point from $E^{-}$. Let $x_{i} \in \Delta \cap E^{-}, i=1,2, x_{1} \neq x_{2}$. Denote by $l$ the maximal number such that both $x_{i}$ belong to the same interval $\delta$ of range $l-1$. So $l>k_{0}$ and there exist two disjoint intervals $\delta_{i}$ of range $l$ such that $x_{i} \in \delta_{i}(i=1,2)$. Inside $\delta$ the first $l-1$ members of series (1) are constants so we have

$$
f_{1}(t)=c+q^{l} r\left(2^{\nu_{l}} t\right)+\omega(t), \quad|\omega(t)|<\frac{1}{9} q^{l} .
$$

Consider two different cases:

(i) $r\left[2^{\nu_{l}}\left(x_{1}-0\right)\right]=-r\left[2^{\nu_{l}}\left(x_{2}-0\right)\right]$.

In this case we have

$$
\left|f_{1}\left(x_{1}-0\right)-f_{1}\left(x_{2}-0\right)\right|>q^{l}
$$

so by (3)

$$
\left|x_{1}-x_{2}\right|^{-\varepsilon}\left|g^{\prime}\left(x_{1}\right)-g^{\prime}\left(x_{2}\right)\right|>g^{l} \cdot 2^{\varepsilon \nu_{l-1}} \text {. }
$$

If $k_{0}$ is large enough and taking (2) into account, this inequality contradicts the condition $g^{\prime} \in C^{\varepsilon}$.

(ii) $r\left[2^{\nu_{l}}\left(x_{1}-0\right)\right]=r\left[2^{\nu_{l}}\left(x_{2}-0\right)\right]($ say , $=1)$.

In this case the intervals $\delta_{i}$ are separated by at least one interval of range $l$, and it is easy to see that the average of $r\left(2^{\nu_{l}} t\right)$ on segment $\left[x_{1}, x_{2}\right]$ does not exceed $2 / 3$. Thus

$$
\left(x_{2}-x_{1}\right)^{-1} \int_{x_{1}}^{x_{2}} f_{1}(t) d t<c+\frac{2}{3} q^{l}+\frac{1}{9} q^{l} .
$$

But $f_{1}\left(x_{1}-0\right)>c+\frac{8}{9} q^{l}$, so

$$
f_{1}\left(x_{1}-0\right)-\left(x_{2}-x_{1}\right)^{-1} \int_{x_{1}}^{x_{2}} f_{1}(t) d t>\frac{1}{9} q^{l} \text {. }
$$


Because of (3), the left-hand side is equal to

$$
\begin{aligned}
g^{\prime}\left(x_{1}\right)-\left(x_{2}-x_{1}\right)^{-1}\left[f\left(x_{2}\right)-f\left(x_{1}\right)\right] & =g^{\prime}\left(x_{1}\right)-\left(x_{2}-x_{1}\right)^{-1}\left(g\left(x_{2}\right)-g\left(x_{1}\right)\right) \\
& \left.=g^{\prime}\left(x_{1}\right)-g^{\prime}(\xi), \quad \xi \in\right] x_{1}, x_{2}[.
\end{aligned}
$$

So

$$
\left|x_{1}-\xi\right|^{-\varepsilon}\left|g^{\prime}\left(x_{1}\right)-g^{\prime}(\xi)\right|>\frac{1}{9} q^{l} 2^{\varepsilon \nu_{l-1}}
$$

and we again have the contradiction with the property of $g$ if $k_{0}$ is large enough.

Thus we obtain that the set $E^{-}$is finite. By similar arguments we get the same on the set $E^{+}$(of right hand accumulation points of $E_{f, g}$ ), and the proof is over.

Remarks. 1. It is clear that by increasing the growth of parameters $\nu_{k}$ we obtain a Lipschitz function $f$ which is impossible to interpolate on any uncountable set by the function $g \in C^{1}(I)$ with the previously given module of continuity of its derivative.

2. It is well known that the Rademacher series and lacunary trigonometric series have the same behavior in many problems and they are used in the same way in the construction of various counterexamples. But, if we replace the Rademacher functions in (1) by harmonics $\cos 2^{\nu_{k}} t$ then $f$ will admit interpolation by a function which is twice smooth. This follows from Theorem 2.

3. A function $f$ is said to be $n$-convex ( $n$-concave) on a set $E \subset \mathbb{R}$ if the $n$th divided difference of $f$,

$$
V\left[f ; x_{0}, x_{1}, \ldots, x_{n}\right]=\sum_{j} \frac{f\left(x_{j}\right)}{\omega^{\prime}\left(x_{j}\right)}, \quad \omega(x)=\prod_{j}\left(x-x_{j}\right),
$$

satisfies the condition $V \geq 0(V \leq 0)$ whenever $x_{0}, x_{1}, \ldots, x_{n}$ are distinct elements of $E$. In [ABLP, p. 677] the following problem is formulated: let $f \in C(I)$ be arbitrary and let $n$ be a positive integer. Does there exist a (nonempty) perfect set $T \subset I$ on which $f$ is either $n$-convex or $n$-concave? The authors noted that for $n=1$ it is known. For $n=2$ this conjecture was proved by Buczolich [Bu]. It turns out, however, that this conjecture is already not true for $n=3$ : The function $f$ from the theorem above is not 3-convex (and 3-concave) on any nonempty perfect set.

In fact, the recurrence formula

$$
V\left[f ; x_{0}, x_{1}, x_{2}, x_{3}\right]=\frac{1}{x_{0}-x_{3}}\left[V\left(f ; x_{0}, x_{1}, x_{2}\right)-V\left(f ; x_{1}, x_{2}, x_{3}\right)\right]
$$

implies that if $f$ is, say, 3-convex on $P$, then $V\left(f ; x_{0}, x_{1}, x_{2}\right)$ is monotone increasing for every argument. So, it is enough to check that the second divided difference of $f$ is unbounded on every perfect $E$. Let us fix the points $x_{1} \neq$ $x_{2} \in E$ which belong to some interval $\Delta$ of range $k$. We assume that $x_{i}$ are dyadical-irrational (it is easy to see that $f_{1}$ is continuous at such points, so $\left.f^{\prime}(x)=f_{1}(x)\right)$.

If the pair $\left(x_{1}, x_{2}\right)$ satisfies condition (ii), then

$$
\left|f^{\prime}\left(x_{1}\right)-\left(x_{2}-x_{1}\right)^{-1}\left[f\left(x_{2}\right)-f\left(x_{1}\right)\right]\right|>\frac{1}{9} q^{l} .
$$


In case (i) we have $\left|f^{\prime}\left(x_{1}\right)-f^{\prime}\left(x_{2}\right)\right|>q^{l}$, so (exchanging the notation if necessary) we can write the same inequality. Now choose the point $x_{0} \in$ $\delta \cap E$ (see above) so close to $x_{1}$ that this inequality holds for $\mid V\left(f ; x_{0}, x_{1}\right)-$ $V\left(f ; x_{1}, x_{2}\right) \mid$ on the left. Thus,

$$
\left|V\left[f ; x_{0}, x_{1}, x_{2}\right]\right|>\frac{1}{9} q^{l}\left|x_{0}-x_{2}\right|^{-1}>\frac{1}{9} q^{l}|\delta|^{-1}=\frac{1}{9} q^{l} 2^{\nu_{l-1}} .
$$

The last term goes to infinity when $k \rightarrow \infty$.

$$
2
$$

Next we see that if $f$ is slightly smoother than in Theorem 1 the possibility of interpolation by smooth functions increases sharply.

Theorem 2. For every $f \in C^{1}(I)$ there exists a function $g \in C^{2}(I)$ which coincides with $f$ on some perfect set.

We need the following lemmas.

Lemma 1 ("about mountaineers"). Let $h \in C[-1,1]$ satisfy the following conditions:

(i) $h(-1)=h(1)=0, h(0)=1 ; h\{]-1,1[\backslash 0\}=] 0,1[$;

(ii) $h$ has only a finite number of extremums;

(iii) all values of $h$ at points of extremums are different.

Then there exist continuous mappings $\varphi:[0,1] \rightarrow[-1,0]$ and $\psi: p[0,1] \rightarrow$ $[0,1]$ such that $\varphi(0)=-1, \psi(0)=1, \varphi(1)=\psi(1)=0$, and $h \circ \varphi \equiv h \circ \psi$.

This lemma allows the next interpretation: two mountaineers starting an ascent by the east and west slopes simultaneously, are always able to reach the top of the mountain in such a way that at every point in time they are at the same altitude. By analyzing the various profiles of the slopes (graph $h$ ) we can see that paths of their moves $(\varphi(t),(h \circ \varphi)(t))$ and $(\varphi(t),(h \circ \psi)(t))$ may have to be very complicated.

Condition (iii) is not essential and is stated to simplify the proof. After this paper was written I was told by N. Alon and M. Laczkovich that this lemma is known (see [GPY]). However, for completeness of presentation I include the proof. Some simplification in the proof is due to $\mathbf{M}$. Gerver.

Proof. We call a "statement" any pair of numbers $s=\left(x_{1}, x_{2}\right)$ with the property

$$
\left.x_{1} \in\right]-1,0\left[, \quad x_{2} \in\right] 0,1\left[, \quad h\left(x_{1}\right)=h\left(x_{2}\right)\right.
$$

and either $x_{1}$ or $x_{2}$ is the point of extremum of $h$.

We also include in the set of statements the pairs $\underline{s}=(-1,1)$ and $\bar{s}=(0,0)$. Let us say that the two statements $s=\left(x_{1}, x_{2}\right)$ and $s^{\prime}=\left(x_{1}^{\prime}, x_{2}^{\prime}\right)\left(s \neq s^{\prime}\right)$ "are connected" if the intervals $] x_{i}, x_{i}^{\prime}[(i=1,2)$ do not contain any points of extremum. It is easy to see that every statement $s$, except $\underline{s}, \bar{s}$, is connected with exactly two different statements, but $\underline{s}$ and $\bar{s}$ only with one. Let us move on the graph $S=\{s\}$ starting from $s_{0}=\underline{s}$, and on every step $k$ going from $s_{k}=\left(x_{1}^{(k)}, x_{2}^{(k)}\right)$ to $s_{k+1}$ which is connected with $s_{k}$ and different from $s_{k-1}$. It is clear that a cycle is impossible (in this case we have $s_{l}=s+k$, $l>k+2$, which means $s_{k}$ is connected with $s_{k-1}, s_{k+1}$, and $s_{l-1}$, which are all different). Since $S$ is finite we unavoidably come to $s_{N}=\bar{s}$. Dividing 
the segment $I=[0,1]$ into $N$ parts, $0=t_{0}<t_{1}<\cdots<t_{N}=1$, suppose $\varphi\left(t_{k}\right)=x_{1}^{(k)}, \psi\left(t_{k}\right)=x_{2}^{(k)}$. Define $\varphi$ on $\left[t_{k-1}, t_{k}\right.$ [ by linear interpolation. On segments $\left[x_{i}^{(k-1)}, x_{i}^{(k)}\right](i=1,2), h$ is strictly monotone and maps them both to the same segment $\subset[0,1]$.

Finally suppose $\psi(t)=\left(h^{-1} \circ h \circ \varphi\right)(t), t \in\left[t_{k-1}, t_{k}\right], h^{-1}(y) \in\left[x_{2}^{(k-1)}, x_{2}^{(k)}\right]$, and we are done.

One can note that this lemma is true for a team of $n$ sportsmen each moving on his slope.

Lemma 2. Let $h \in C[a, d], a<b<c<d$, and suppose that

$$
h(a)=h(c)=\max _{t \in[a, d]} h(t) ; \quad h(b)=h(d)=\min _{t \in[a, d]} h(t) .
$$

Then there exist the points $x_{1} \in[a, b], x_{2} \in[c, d]$ such that

$$
h\left(x_{1}\right)=h\left(x_{2}\right)=\left(x_{2}-x_{1}\right)^{-1} \int_{x_{1}}^{x_{2}} h(t) d t .
$$

First, suppose that $h(] a, b[\cup] c, d[) \subset] h(b), h(c)[$ and that (ii), (iii) of the previous lemma are fulfilled on $] a, d[$. Then, this lemma (after an evident change of variable, which carries $[a, b]$ on $[0,1],[c, d]$ on $[0,-1]$, and $[h(b), h(c)]$ on $[0,1])$ gives the mappings $\varphi:[0,1] \rightarrow[a, b]$ and $\psi:[0,1] \rightarrow$ $[c, d]$ with the following properties: $\varphi(0)=a, \varphi(1)=b, \psi(0)=c, \psi(1)=$ $d ; h \circ \varphi \equiv h \circ \psi$. Suppose

$$
J(t)=[\psi(t)-\varphi(t)]^{-1} \int_{\varphi(t)}^{\psi(t)} h(\tau) d \tau \quad(t \in[0,1]) .
$$

Evidently $J(0)<(h \circ \varphi)(0), J(1)>(h \circ \varphi)(1)$. By continuity we can choose $\bar{t}$ such that

$$
J(\bar{t})=(h \circ \varphi)(\bar{t}) \quad(=(h \circ \psi)(\bar{t})) .
$$

Taking $x_{1}=\varphi(\bar{t}), x_{2}=\psi(\bar{t})$ we obtain what we need.

If the "mountaineers lemma" is not directly applicable we approximate $h$ by $h_{n}$ uniformly (say, with error $<1 / n$ ) in such a way that for $h_{n}$ conditions (ii), (iii), and (1) hold and we find the points $x_{1}^{(n)} \in[a, b], x_{2}^{(n)} \in[c, d]$ with

$$
h_{n}\left(x_{1}^{(n)}\right)=h_{n}\left(x_{2}^{(n)}\right)=\left(x_{2}^{(n)}-x_{1}^{(n)}\right)^{-1} \int_{x_{1}^{(n)}}^{x_{2}^{(n)}} h_{n}(t) d t .
$$

Taking a subsequence, we can assume that $x_{i}^{(n)} \rightarrow x_{i}(i=1,2)$. Clearly we obtain (2).

The function $h$ is said to be nowhere monotone if it has no interval of monotonicity.

Lemma 3. If $h$ is continuous and nowhere monotone on segment $I_{0}$, then we can choose two points $x_{1} \neq x_{2}$ in $I_{0}$ with condition (2).

Denote by $t_{1}, t_{2}$ the points of minimal and maxmimal values of $h$ on $I_{0}$ (say, $t_{1}<t_{2}$ ). We take some point of local minimum $t_{3} \in\left[t_{1}, t_{2}\right]$ and denote by $b$ the point of maximal value $h$ on $\left[t_{1}, t_{3}\right]$. Of course $b<t_{3}$, so the level set of $h$ containing $b$ has a nonempty intersection with the segment $\left[t_{3}, t_{2}\right]$. 
Denote by $d$ the closest point to $b$ of this intersection. Let $c$ be the point of minimal value $h$ on segment $[b, d]$, so $b<c<d$. Finally denote by $a$ the closest solution to $b$ of the equation $h(t)=h(c), t_{1} \leq t<b$. Now it is clear that all conditions of the previous lemma are fulfilled.

Let us now return to the proof of Theorem 2. It is well known that if $f \in$ $C^{1}\left(I_{0}\right)$ and $f^{\prime}$ is differentiable a.e. then there exists a function $g \in C^{2}\left(I_{0}\right)$ which coincides with $f$ on a set of positive Lebesgue measure (see, e.g., [Fe, Theorem 3.1.5]). So we need only consider the case where $f^{\prime}$ is nowhere monotone on $I$. Using the previous lemma we find inside $I$ two points $a_{1}, a_{2}$ such that the equality,

$$
f^{\prime}\left(a_{1}\right)=f^{\prime}\left(a_{2}\right)=\left(a_{2}-a_{1}\right)^{-1}\left[f\left(a_{2}\right)-f\left(a_{1}\right)\right],
$$

is true which means that the graph $f$ has a common tangent at points with abscissa $a_{i}(i=1,2)$. For small $\delta_{1}>0$ denote by $\Delta_{i}$ the closed $\delta_{1}$-neighborhoods of the points $a_{i}$ and call them segments of range 1 . Thus for every $x_{i} \in \Delta_{i}(i=1,2)$ we have

$$
\left|f^{\prime}\left(x_{1}\right)-f^{\prime}\left(x_{2}\right)\right| \leq\left|f^{\prime}\left(x_{1}\right)-f^{\prime}\left(a_{1}\right)\right|+\left|f^{\prime}\left(x_{2}\right)-f^{\prime}\left(a_{2}\right)\right| \leq 2 \omega\left(f^{\prime} ; \delta_{1}\right)
$$

$(\omega(\varphi ; \delta)$ is the modulus of continuity of the function $\varphi)$, and also

$$
\begin{aligned}
& \left|f\left(x_{2}\right)-f\left(x_{1}\right)-f^{\prime}\left(x_{1}\right)\left(x_{2}-x_{1}\right)\right| \\
& \left.\quad=\mid f^{\prime}\left(x_{2}\right)-f\left(a_{2}\right)\right]-\left[f\left(x_{1}\right)-f\left(a_{1}\right)\right]-\left(f^{\prime}\left(x_{1}\right)-f^{\prime}\left(a_{1}\right)\right)\left(x_{2}-x_{1}\right) \\
& \quad \leq 4\left\|f^{\prime}\right\|_{C(I)} \delta_{1}+\omega\left(f^{\prime} ; \delta_{1}\right)\left|x_{2}-x_{1}\right| .
\end{aligned}
$$

It is clear that choosing $\delta_{1}$ small enough we have

$$
\begin{gathered}
\left|f^{\prime}\left(x_{1}\right)-f^{\prime}\left(x_{2}\right)\right|<\frac{1}{2}\left|x_{1}-x_{2}\right| ; \\
\left|f\left(x_{2}\right)-f\left(x_{1}\right)-f^{\prime}\left(x_{1}\right)\left(x_{2}-x_{1}\right)\right|<\frac{1}{2}\left|x_{1}-x_{2}\right|^{2},
\end{gathered}
$$

and by the same method

$$
\left|f\left(x_{1}\right)-f\left(x_{2}\right)-f^{\prime}\left(x_{2}\right)\left(x_{2}\right)\left(x_{1}-x_{2}\right)\right|<\frac{1}{2}\left|x_{1}-x_{2}\right|^{2} .
$$

Then we apply Lemma 3 on each segment $\Delta_{i}$ and find inside corresponding pairs of points $\left(a_{11}, a_{12}\right)$ and $\left(a_{21}, a_{22}\right)$ such that

$$
f^{\prime}\left(a_{i 1}\right)=f^{\prime}\left(a_{i 2}\right)=\left(a_{i 2}-a_{i 1}\right)^{-1}\left[f\left(a_{i 2}\right)-f\left(a_{i 1}\right)\right] \quad(i=1,2) .
$$

This enables us, as above, to choose small $\delta_{2}>0$ so that in closed $\delta_{2}$-neighborhoods-segments of range $2-\Delta_{i j} \subset \Delta_{i}(i, j=1,2)$ we have

$$
\begin{gathered}
\left|f^{\prime}\left(x_{1}\right)-f^{\prime}\left(x_{2}\right)\right|<\frac{1}{4}\left|x_{1}-x_{2}\right|, \\
\left|f\left(x_{2}\right)-f\left(x_{1}\right)-f^{\prime}\left(x_{1}\right)\left(x_{2}-x_{1}\right)\right|<\frac{1}{4}\left|x_{2}-x_{1}\right|^{2},
\end{gathered}
$$

and symmetrically

$$
\left|f\left(x_{1}\right)-f\left(x_{2}\right)-f^{\prime}\left(x_{2}\right)\left(x_{1}-x_{2}\right)\right|<\frac{1}{4}\left|x_{1}-x_{2}\right|^{2}
$$

for every $x_{j} \in \Delta_{i j}, i, j=1,2$.

Continuing this process we finally get a perfect $E$ (intersection of compacts $E_{k}$ which are the union of the segments of range $k$ ) so that for $x, y \in E$ the following properties hold:

$$
\left|f^{\prime}(x)-f^{\prime}(y)\right|=o(|x-y|), \quad\left|f(x)-f(y)-f^{\prime}(y)(x-y)\right|=o\left(|x-y|^{2}\right)
$$


with uniform $o$. Finally the Whitney theorem [St] allows the extension of the function $\left.f\right|_{E}$ to some $g \in C^{2}(I)$ (with $\left.g^{\prime \prime}\right|_{E} \equiv 0$ ) and the theorem is proven.

We will use the notations for a given function $\psi$ and points $y \neq x$ :

$$
\begin{aligned}
& T_{1}(\psi ; x, y)=|\psi(x)-\psi(y)| ; \\
& T_{2}(\psi ; x, y)=\left|\psi(x)-\frac{1}{y-x} \int_{x}^{y} \psi(t) d t\right| \\
& T_{3}(\psi ; x, y)=\left|\frac{1}{y-x} \int_{x}^{y} \psi(t) d t-\frac{2}{(y-x)^{2}} \int_{x}^{y}(y-t) \psi(t) d t\right| .
\end{aligned}
$$

The key point of this section is the following proposition.

Theorem 3. For every $\beta, 0<\beta<1$, there exists a function $\psi\left(=\psi_{\beta}\right) \in C^{\beta}(I)$ such that for every point $x \neq y$ belonging to $I$ the inequality

(*) $\quad T(\psi ; x, y) \equiv T_{1}(\psi ; x, y)+T_{2}(\psi ; x, y)+T_{3}(\psi ; x, y)>c(\beta)|x-y|^{\beta}$

holds, where $c(\beta)>0$.

We note that all terms in $T_{j}(1 \leq j \leq 3)$ are averages of $\psi$ over some positive nieasures with unit mass, supported on $[x, y]$, so the inverse estimate is evident.

Lemma 4. Let $\varphi$ be a function defined on the interval [0, 4[ by the equality

$$
\varphi(t)= \begin{cases}1, & 0 \leq t<1, \\ -1, & 1 \leq t<2, \\ 2, & 2 \leq t<3 \\ -2, & 3 \leq t<4\end{cases}
$$

and periodically extended on $\mathbb{R}$. Then

(i) for every $u, v, v \notin \mathbb{Z}$, which are separated by a segment $[a, a+1], a \in$ $\mathbb{Z}$, the inequality $T_{2}(\varphi ; u, v)>\frac{1}{3}$ holds

(ii) for every integer $a, b, b>a+1$, the inequality $T_{3}(\varphi ; a, b) \geq$ $\frac{1}{3}(b-a)^{-1}$ holds

(iii) if $0<\lambda<10^{-4},|u-a|<\lambda,|v-b|<\lambda, a, b \in \mathbb{Z}, b>a+1$, then $T_{3}(\varphi ; u, v)>\frac{1}{4}(v-u)^{-1}$.

One can check part (i) directly. To prove (ii) we denote

$$
\phi(t)=\int_{0}^{t} \varphi(\tau) d \tau .
$$

After integration by parts, the inequality in (ii) can be written in the form

$$
\left|\int_{a}^{b} \phi d u-\frac{1}{2}(b-a)(\phi(a)+\phi(b))\right| \geq \frac{1}{6}(b-a)
$$

which gives an estimate from below of the error of quadrature of $\phi$ by linear interpolation. If $b=a+2$ we check this estimate directly. If not it is easy to see that the average of $\phi$ over $[a, b]$ is bounded in limits $\left[\frac{2}{3}, \frac{5}{6}\right]$, but the value $\frac{1}{2}(\phi(a)+\phi(b))$ is half of an integer. 
By estimating partial derivatives $\partial T_{3} / \partial u, \partial T_{3} / \partial v$ we obtain that if $u, v$ change in $\lambda$-neighborhoods of $a, b$ the corresponding difference of $T_{3}$ does not exceed $\frac{1}{10}(b-a)^{-1}$, so (ii) implies (iii).

Now we are going over to the construction of function $\psi$.

1. Denote by $\varphi_{\lambda}$ the function $\varphi$ from the lemma after linear interpolation in $\lambda$-neighborhoods of integers. $\varphi$ has a period equal to 4 and integral over the period equal to zero. So the estimates are evident for any $u, v \in \mathbb{R}$ :

$$
\left|\int_{u}^{v} \varphi_{\lambda} d t\right|<2, \quad\left|\int_{u}^{v}\left(\varphi_{\lambda}-\varphi\right) d t\right|<2 \lambda
$$

2. We fix an integer $q$ such that

$$
\min \left\{q, q^{1 / \beta-1}\right\}>10^{6}
$$

and define by induction a sequence of continuous piecewise linear functions $\psi_{n}$. On the first step we call $I=[0,1]$ a segment of range zero and put

$$
\nu(I)=\left[\frac{1}{3} q^{1 / \beta}\right] \quad([\alpha] \text { is the integer part of } \alpha),
$$

$\theta=\theta(I)$ is an affine mapping $I \rightarrow[0, \nu]$. Now we take $\varphi$ from Lemma 4 and denote by $\psi_{1}$ the function $q^{-2} \varphi \circ \theta$ after linear interpolation in $\delta_{0}$ neighborhoods of disconnection points (with $\delta_{0}=q^{1-1 / \beta}$ ).

Segments of constancy of $\psi_{1}$ we call segments of range one; adjacent intervals we call splits of range one.

Let us assume that we have already defined a function $\psi_{n-1} \in C(I)$ and a partition of the segment $I$ into a collection of closed intervals $s$ (called segments of range $n-1$ ) and separating them open intervals $\mathfrak{a}$ (called splits of range $n-1)$. Denote by $S_{n-1}$ and $\mathfrak{A}_{n-1}$ respectively the set of these intervals.

Suppose that the following induction assumption is fulfilled:

(3) $\psi_{n-1}$ is a constant on any $s \in S_{n-1}$ and $q^{-(n-1) / \beta} \leq|s| \leq 100 q^{-(n-1) / \beta}$,

(4) $\psi_{n-1}$ is a linear function on any $\mathfrak{a} \in \mathfrak{A}_{n-1}$ and

$$
|\mathfrak{a}|=2 \delta_{n-1} \equiv q^{-n / \beta+1} ; \quad 2 q^{-n} \leq\left|\Delta_{\mathfrak{a}} \psi_{n-1}\right| \leq 10 q^{-n}
$$

$\left(\Delta_{\mathfrak{a}} \psi=\psi\left(\tau^{\prime}\right)-\psi(\tau)\right.$ for $\left.\mathfrak{a}=\right] \tau, \tau^{\prime}[)$.

Now to define a function $\psi_{n}$ we divide every split $\mathfrak{a} \in \mathfrak{A}_{n-1}$ into $\mu$ equal parts:

$$
\mu \quad(=\mu(\mathfrak{a}))=\left[\frac{1}{10}\left|\Delta_{\mathfrak{a}} \psi_{n-1}\right| q^{n+1}\right]+1,
$$

and then we divide every segment $s=\left[d, d^{\prime}\right] \in S_{n-1}$ into $\nu$ equal parts:

$$
\nu \quad(=\nu(s))=\left[\frac{1}{3}|s| q^{n / \beta}\right] .
$$

All points of the partition (also including ends of the interval $\mathfrak{a} \in \mathfrak{A}_{n-1}$ ) we surround by $\delta_{n}$-neighborhoods, which are splits of range $n$. All adjacent segments we call segments of range $n$. It is easy to check that induction assumptions related to lengths of splits and segments are fulfilled on the $n$-step.

Then we define $\psi_{n}$ on segments of range $n$ belonging to any $\left.\mathfrak{a}=\right] \tau, \tau^{\prime}[\epsilon$ $\mathfrak{A}_{n-1}$ :

$\psi_{n}(t)=\psi_{n-1}\left(\tau+\frac{i-\frac{1}{2}}{\mu}|\mathfrak{a}|\right) \quad$ for $\tau+\frac{i-1}{\mu}|\mathfrak{a}|+\delta_{n}<t<\tau+\frac{i}{\mu}|\mathfrak{a}|-\delta_{n}(1 \leq i \leq \mu)$. 
Further for any $s \in S_{n-1}$ we consider the affine mapping $\theta\left(=\theta_{s}\right)$ which transforms $s$ to the segment $[0, \nu(s)]$. Let

$$
\lambda \quad(=\lambda(s))=\delta_{n} \nu /|s| .
$$

(2) implies that $\lambda<10^{-4}$. It is clear that $\theta$ maps splits belonging to $s$ onto $\lambda$-neighborhoods of integers.

Set

$$
\psi_{n}(t)=\psi_{n-1}(t)+q^{-(n+1)}(\varphi \circ \theta)(t) ; \quad t \in \bigcup_{s^{\prime} \in S_{n} ; s^{\prime} \subset s} .
$$

(7) and (9) define $\psi_{n}$ on every segment of range $n$ by some constant. To conclude, we define $\psi_{n}$ on splits of range $n$ by linear interpolation. It is easy to check that the estimate of differences in (4) is preserved for $\psi_{n}, \mathfrak{a} \in \mathfrak{A}_{n}$.

The construction above immediately implies that

$$
\left\|\psi_{n}-\psi_{n-1}\right\|_{C(I)}<10 q^{-(n+1)},
$$

so $\psi_{n}$ converge uniformly to a function $\psi$ and

$$
\left\|\psi-\psi_{n-1}\right\|_{C(I)}<11 q^{-(n+1)} .
$$

We claim that $\psi \in C^{\beta}(I)$. In fact, if

$$
q^{1-(n+1) / \beta} \leq|x-y|<q^{-n / \beta+1},
$$

then, by (3), (4) we have

$$
\left|\psi_{n-1}(x)-\psi_{n-1}(y)\right|<10 q^{-n},
$$

and (11) implies the same inequality (with another constant) for $\psi$.

We note that for every $s=\left[d, d^{\prime}\right] \in S_{n-1}$ the function

$$
\varphi^{s}(t)=\left(\psi_{n}-\psi_{n-1}\right)(t) \quad(t \in s)
$$

satisfies conditions

$$
\begin{gathered}
\varphi^{s}(t)=q^{-(n+1)}\left(\varphi_{\lambda} \circ \theta\right)(t), \quad d+\delta_{n} \leq t \leq d^{\prime}-\delta_{n}, \\
\left|\varphi^{s}(t)\right| \leq \frac{7}{2} q^{-(n+1)}, \quad t \in s .
\end{gathered}
$$

3. We also need the integral estimates

$$
\begin{aligned}
& \frac{1}{|J|}\left|\int_{J}\left(\psi-\psi_{n-1}\right) d t\right|<360 q^{-(n+1)-1 / \beta} \\
& \frac{2}{|J|^{2}}\left|\int_{J}(y-t)\left(\psi-\psi_{n-1}\right) d t\right|<710 q^{-(n+1)-1 / \beta}
\end{aligned}
$$

for every segment $J=[x, y] \subset I,|J|>q^{-(n-1) / \beta}$.

To prove this, first note that (7) implies that the integral of $\psi_{n}-\psi_{n-1}$ over every segment and split of range $n$, belonging to any split $\mathfrak{a} \in \mathfrak{A}_{n-1}$, equals to zero. So for any interval $j \subset \mathfrak{a}$ we have, using (10), (4), (5),

$$
\left|\int_{j}\left(\psi_{n}-\psi_{n-1}\right) d t\right| \leq\left\|\psi_{n}-\psi_{n-1}\right\|_{C(I)} \cdot 2 \frac{|\mathfrak{a}|}{\mu}<100 q^{-(n+1)-n / \beta} .
$$

If the interval $j \subset s \subset S_{n-1}$ we obtain from (12), (6), and (1)

$$
\left|\int_{j}\left(\psi_{n}-\psi_{n-1}\right) d t\right| \leq q^{-(n+1)}\left(2 \frac{|s|}{\nu}+7 \delta_{n}\right)<7 q^{-(n+1)-n / \beta} .
$$


For any interval $J \subset I$ the number of segments $s \in S_{n-1}$, which have nonempty intersection with $J$ does not exceed $|J|_{q}^{(n-1) / \beta}+2$, so we have from (15), (16)

$$
\left|\int_{J}\left(\psi_{n}-\psi_{n-1}\right) d t\right|<117 q^{-(n+1)-n / \beta}\left(|J| q^{(n-1) / \beta}+2\right) .
$$

Replacing $n$ by $k$ we obtain after summation over $k \geq n$,

$$
\left|\int_{J}\left(\psi-\psi_{n-1}\right) d t\right|<118 q^{-(n+1)-n / \beta}\left(|J| q^{(n-1) / \beta}+2\right) .
$$

To estimate $\int_{J}(y-t)\left(\psi-\psi_{n-1}\right)(t) d t$ we integrate by parts and use the previous inequality for $\int_{x}^{t}\left(\psi-\psi_{n-1}\right) d \tau(t \in J)$. Thus we obtain

$$
\left|\int_{J}(y-t)\left(\psi-\psi_{n-1}\right)(t) d t\right|<118 q^{-(n+1)-n / \beta}\left(|J| q^{(n-1) / \beta}+2\right)|J| .
$$

For $|J|>q^{-(n-1) / \beta} \quad(17)$ and (18) give (13) and (14).

4. Let $s \in S_{n-1}$, and let $\bar{s}$ be the union of $s$ with two adjacent splits $\mathfrak{a}_{i} \in \mathfrak{A}_{n-1}$ (with one split, if $s$ is an end-segment; $\bar{s}=I$ if $n=1$ ).

We prove the following

Proposition. If $x, y \in \bar{s}$ are separated by a split of range $n$ then at least one of the following conditions holds:

$$
\begin{aligned}
& T_{1}\left(\psi_{n} ; x, y\right)+T_{2}\left(\psi_{n} ; x, y\right)>\frac{1}{20} \cdot q^{-(n+1)}, \\
& T_{3}\left(\psi_{n} ; x, y\right)>6 \cdot 10^{-3} \cdot q^{-(n+1)-1 / \beta} \text { and }|x-y|>q^{-n / \beta} .
\end{aligned}
$$

Denote by $\mathfrak{a}_{i}^{0}$ the split of range $n$ which intersects with $s$ and $\mathfrak{a}_{i}$.

(I) Let at least one point $x, y$ lie outside $s$.

It is easy to check from point 2 above that

4.1. $\psi_{n}$ is monotone on every interval $\mathfrak{a}_{i} \cup \mathfrak{a}_{i}^{0}$ and differences $\left|\Delta_{\mathfrak{a}} \psi_{n}\right|>$ $q^{-(n-1)} \quad \forall \mathfrak{a} \in \mathfrak{A}_{n}, \mathfrak{a} \subset \mathfrak{a}_{i}$.

4.2. If $x \in \mathfrak{a}_{i}, y \in s \backslash \mathfrak{a}_{i}^{0}$ then $T_{1}\left(\psi_{n} ; x, y\right)>\frac{1}{4} q^{-(n+1)}$.

So it is enough to consider the case when $x \in \mathfrak{a}_{1}$ and $y \in \mathfrak{a}_{2}$. We can assume also

$$
T_{1}\left(\psi_{n} ; x, y\right)<\frac{1}{20} q^{-(n+1)} .
$$

Let, e.g., $x<y, \psi_{n}$ increase on $\mathfrak{a}_{1}$. Then it follows from the above that $\psi_{n}$ decreases on $\mathfrak{a}_{2}$. We write

$$
\begin{array}{lll}
\psi_{n}(t)>\psi_{n}(x)+\frac{1}{4} q^{-(n+1)}, & & t \in s \backslash\left(\mathfrak{a}_{1}^{0} \cup \mathfrak{a}_{2}^{0}\right), \\
\psi_{n}(t)>\psi_{n}(x), & & t \in \mathfrak{a}_{1} \cup \mathfrak{a}_{1}^{0}, x<t, \\
\psi_{n}(t)>\psi_{n}(y)>\psi_{n}(x)-\frac{1}{20} q^{-(n+1)}, & & t \in \mathfrak{a}_{2} \cup \mathfrak{a}_{2}^{0}, t<y .
\end{array}
$$

Thus we have

$$
\frac{1}{y-x} \int_{x}^{y} \psi_{n} d t>\psi_{n}(x)+\frac{1}{4} q^{-(n+1)} \frac{|s|-2 \delta_{n}}{|s|+2 \delta_{n-1}}-\frac{1}{20} q^{-(n+1)} \frac{2 \delta_{n-1}+2 \delta_{n}}{|s|-2 \delta_{n}} .
$$

Using (3), (4) we obtain

$$
T_{2}\left(\psi_{n} ; x, y\right)>\frac{1}{10} q^{-(n+1)} .
$$

(II) Let $x, y \in s$. First we consider the case when 
(IIi) $x$ belongs to a segment of range $n$.

Taking into account that $x, y$ are separated by a split of range $n$ and using (21), we see that points $u=\theta(y), v=\theta(x)$ satisfy the conditions of Lemma 4(i). Further we note from (12) and (1),

$$
\left|\int_{j}\left[\left(\varphi^{s} \cdot \theta^{-1}\right)(\tau)-q^{-(n+1)} \varphi(\tau)\right] d \tau\right|<10 \lambda q^{-(n+1)}
$$

for every interval $j \subset[0, \nu]$. Moreover

$$
\left(\varphi^{s} \circ \theta^{-1}\right)(\theta x)=\varphi(\theta x)
$$

so

$$
\begin{aligned}
T_{2}\left(\psi_{n} ; x, y\right) & =T_{2}\left(\varphi^{s} ; x, y\right)=T_{2}\left(\varphi^{s} \circ \theta^{-1} ; \theta(x), \theta(y)\right) \\
& >q^{-(n+1)} T_{2}(\varphi ; \theta(x), \theta(y))-10 \lambda|\theta(y)-\theta(x)|^{-1} q^{-(n+1)},
\end{aligned}
$$

and using Lemma $4(i)$ we obtain

$$
T_{2}\left(\psi_{n} ; x, y\right)>\frac{1}{4} q^{-(n+1)} .
$$

If $y$ belongs to a segment of range $n$ we obtain the same estimate for $T_{2}\left(\psi_{n} ; y, x\right)$ and then (21) implies

$$
T_{2}\left(\psi_{n} ; x, y\right)>\frac{1}{5} q^{-(n+1)} .
$$

To conclude, let

(IIii) $x, y$ belong to splits of range $n$.

We have, from (22) after integration by parts,

$$
\left|\int_{u}^{v}(v-\tau)\left[\left(\varphi^{s} \circ \theta^{-1}\right)(\tau)-q^{-(n+1)} \varphi(\tau)\right] d \tau\right|<10 \lambda q^{-(n+1)}|u-v|,
$$

so

$$
T_{3}\left(\varphi^{S} \circ \theta^{-1}-q^{-(n+1)} \varphi ; u, v\right)<10 \lambda q^{-(n+1)}|u-v|^{-1} .
$$

We can see that points $u=\theta(y), v=\theta(x)$ satisfy the conditions of Lemma 4(iii). So, taking into account that $T_{3}$ is invariant with respect to affine transform, we obtain

$$
\begin{aligned}
T_{3}\left(\psi_{n} ; x, y\right) & =T_{3}\left(\varphi^{s} ; x, y\right)=T_{3}\left(\varphi^{s} \circ \theta^{-1} ; u, v\right) \\
& >\left(\frac{1}{4}-10 \lambda\right) q^{-(n+1)}|u-v|^{-1}>\frac{1}{5} q^{-(n+1)} \nu^{-1},
\end{aligned}
$$

and using (6) and (3) we have

$$
T_{3}\left(\psi_{n} ; x, y\right)>6 \cdot 10^{-3} q^{-(n+1)-1 / \beta} .
$$

Moreover $x, y$ do not belong to the same split of range $n$, so $|x-y|>q^{-n / \beta}$.

The proposition is proved.

5. Now we immediately finish the proof of Theorem 3. In fact, for every $x \neq y \in I$ we can find a number $n$ such that conditions of the proposition are fulfilled.

If (19) holds we have from (11) and (2),

$$
T_{1}(\psi ; x, y)+T_{2}(\psi ; x, y)>\frac{1}{20} q^{-(n+1)}-2\left\|\psi-\psi_{n}\right\|_{C(I)}>q^{-(n+2)} .
$$

If not we obtain from (20), (13), (14),

$$
T_{3}(\psi ; x, y)>6 \cdot 10^{-3} q^{-(n+1)-1 / \beta}-T_{3}\left(\psi-\psi_{n} ; x, y\right)>q^{-(n+2)-1 / \beta} .
$$


Anyway, since $|x-y|<|s|+4 \delta_{n-1}<q^{-(n-2) / \beta}$, we conclude that $(*)$ is true with $c(\beta)=q^{-4-1 / \beta}$.

In an analogous way (and even slightly easier, because we do not need to consider the smoothness of $\psi$ ) one can prove

Theorem $3^{\prime}$. There exists a function $\psi \in C(I)$ such that

$$
T(\psi ; x, y)>c(\delta)|x-y|^{\delta} \quad(x \neq y \in I)
$$

for every $\delta>0$ with some constant $c(\delta)>0$.

Corollary. Let $\alpha \geq 2$ and $k=[\alpha]$. Then there exists a function $f \in C^{\alpha}(I)$ such that if $g \in C^{\gamma}(I)$ with some $\gamma>\alpha(\gamma \leq k+1)$ then the number $\nu(f, g)$ of points in which $f$ and $g$ have the contact of order $k$ is finite and satisfy the estimate

$$
\gamma_{f, g}<c(\alpha, \gamma) \cdot\left\|g^{(k)}\right\|_{C^{\gamma-k}(I)}^{2 /(\gamma-\alpha)}
$$

(by $\|g\|_{C^{\delta}(I)}, 0<\delta<1$, we mean the Hölder constant

$$
\left.\sup _{x \neq y}|g(x)-g(y)||x-y|^{-\delta}\right) .
$$

We start from the function $\psi$ equals $\psi_{\alpha-k}$ (Theorem 3) if $\alpha>k$ and $\psi$ from Theorem $3^{\prime}$ if $\alpha=k$. Let

$$
f(x)=\int_{0}^{x} \cdots \int_{0}^{u_{1}} \psi(t) d t d u_{1} \cdots d u_{k-1} .
$$

Clearly $f \in C^{\alpha}$ and $(*)$ or $(* *)$ implies

$$
T\left(f^{(k)} ; x, y\right)>c \cdot|x-y|^{(\alpha+\gamma) / 2-k} \quad(\forall x \neq y \in I) .
$$

This inequality can be rewritten in the following way:

$$
\begin{aligned}
& \left|f^{(k)}(x)-f^{(k)}(y)\right|+\left|f^{(k)}(x)-\frac{1}{y-x}\left[f^{k-1}(y)-f^{(k-1)}(x)\right]\right| \\
& +\mid \frac{1}{y-x}\left[f^{(k-1)}(y)-f^{(k-1)}(x)\right] \\
& -\frac{2}{(y-x)^{2}}\left[f^{(k-2)}(y)-f^{(k-2)}(x)-(y-x) f^{(k-1)}(x)\right] \\
& >c \cdot|x-y|^{(\alpha+\gamma) / 2-k} \text {. }
\end{aligned}
$$

If $g$ has a contact of order $k$ at $x$ and $y$ we have the same inequality for $g$. So by the Taylor formula we obtain

$$
\left|g^{(k)}(x)-g^{(k)}(y)\right|+\left|g^{(k)}(x)-g^{(k)}(\xi)\right|+\left|g^{(k)}(\xi)-g^{(k)}(\eta)\right|>c|x-y|^{(\alpha+\gamma) / 2-k}
$$

for some $\xi, \eta$ belonging to the interval $] x, y[$. But the left side does not exceed $3\left\|g^{(k)}\right\|_{C^{\gamma-k}(I)}|x-y|^{\gamma-k}$, so

$$
|x-y|>c^{\prime}\left\|g^{(k)}\right\|^{-2(\gamma-\alpha)} \text {. }
$$

This implies (23).

Now we immediately see that the following theorem is true. 
Theorem 4. For every $\alpha \geq 2$ there exists a function $f \in C^{\alpha}(I)$ such that if $g \in C^{\gamma}(I), \gamma>\alpha$, then the set $E_{f, g}$ is at most countable with only finite number of accumulation points.

It is enough to note that at every accumulation point we have a contact of order $k=[\alpha]$.

We note to conclude that Theorems 1 and 4 cannot be obtained by categories arguments. The properties of functions we constructed are not typical in the corresponding functional spaces in the sense of Baire categories. More exactly, we say a function $f \in Z$ if there exists a function $g \in C^{\infty}(I)$ which coincides with $f$ on some perfect compact.

Proposition. For every integer $k \geq 0$ the set $Z \cap C^{k}(I)$ is residual in the space $C^{k}(I)$.

This means that the complement is a countable union of nowhere dense sets. We give a sketch of the proof.

Lemma 5. Suppose that $f \in C^{k}(I)$ and that for every segment $J \subset I$ one can find a segment $\Delta \subset J$ with the property

$$
\| f^{(k)}(x)-\text { Const } \|_{C(\Delta)}<e^{-1 /|\Delta|} .
$$

Then $f \in Z$.

An inductive process like that which we applied in Theorem 2 enables the use of (24) to construct a perfect set $E$ such that the following equalities are fulfilled:

$$
\begin{aligned}
f^{(k)}(y) & =f^{(k)}(x)+o\left(|y-x|^{l}\right), \\
f^{(k-1)}(y) & =f^{(k-1)}(x)+f^{(k)}(x)(y-x)+o\left(|y-x|^{l+1}\right), \\
& \vdots \\
f(y) & =f(x)+f^{\prime}(x)(y-x)+\cdots+f^{(k)}(x)(y-x)^{k}+o\left(|y-x|^{l+k}\right)
\end{aligned}
$$

for each $l=1,2, \ldots$ with $o$ uniform with respect to $x, y \in E$ for fixed $l$. The Whitney theorem gives an extension $\left.f\right|_{E}$ to the function $g \in C^{\infty}(I)$ (with $\left.\left.g^{(k+l)}\right|_{E}=0\right)$.

Now to prove the proposition it is enough to note that for fixed $J$ the set of functions $f$ which allows the choice of $\Delta=\Delta(f)$ with estimate (24) is open and dense in $C^{k}(I)$.

\section{REFERENCES}

[ABLP] S. Agronsky, A. M. Bruckner, M. Laczkovich, and D. Preiss, Convexity conditions and intersections with smooth functions, Trans. Amer. Math. Soc. 298 (1985), 659-677.

[Ag] S. Agronsky, Intersections of continuous functions with families of smooth functions, Real Anal. Exchange 10 (1984/85), 25-30.

[Br] J. Brown, Differentiable restrictions of real functions, Real Anal. Exchange 13 (1987/88), 44-45.

[Bu] Z. Buczolich, Sets of convexity of continuous function, Acta Math. Hungar. 52 (1988), 291303.

[Ca] H. Cartan, Collected works, Springer-Verlag, Berlin and New York, 1979.

[Fe] H. Federer, Geometric measure theory, Springer, New York, 1969. 
[GPY] J. Goodman, J. Pach, and C. Yap, Mountain climbing, ladder moving, and the ring-width of a polygon, Amer. Math. Monthly 96 (1989), 494-510.

[La] M. Laczkovich, Differentiable restrictions of continuous functions, Acta Math. Hungar. 44 (1984), 355-360.

[Ol] A. Olevskii, Some interpolation problems in real and harmonic analysis, Real Anal. Exchange 16 (1990/91), 363-372.

[Sc] The Scottish Book, Mathematics from the Scottish café, Birkhäuser, Basel and Boston, 1981.

[St] E. Stein, Singular integrals and differentiability properties of functions, Princeton Univ. Press, Princeton, NJ, 1970.

[U] S. Ulam, A collection of mathematical problems, Interscience, New York, 1960.

[Z] Z. Zahorski, Sur l'ensemble des points singuliére d'une fonction d'une variable réele admettand des dérivées de tous les ordres, Fund. Math. 34 (1947), 183-245.

School of Mathematical Sciences, Raymond and Beverly Sackler, Faculty of Exact Sciences, Tel Aviv University, Tel Aviv 69978, Israel

E-mail address: olevskii@math.tau.ac.il 